\title{
A Sub-optimal Algorithm to Synthesize Control Laws for a Network of Dynamic Agents
}

\author{
V. Gupta, B. Hassibi and R. M. Murray \\ Division of Engineering and Applied Science \\ California Institute of Technology \\ \{gupta,hassibi,murray\}@caltech.edu
}

\begin{abstract}
We study the synthesis problem of an LQR controller when the matrix describing the control law is constrained to lie in a particular vector space. Our motivation is the use of such control laws to stabilize networks of autonomous agents in a decentralized fashion; with the information flow being dictated by the constraints of a pre-specified topology. In this paper, we consider the finite-horizon version of the problem and provide both a computationally intensive optimal solution and a sub-optimal solution that is computationally more tractable. Then we apply the technique to the decentralized vehicle formation control problem and show that the loss in performance due to the use of the sub-optimal solution is not huge; however the topology can have a large effect on performance.
\end{abstract}

\section{INTRODUCTION AND MOTIVATION}

Control of dynamic agents coupled to each other through an information flow network has emerged as a topic of major interest in recent years. Such a setting can be used to model many real-life situations, such as air traffi c control, satellite clusters, swarms of robots, UAV formations, and potentially such applications as the Internet. Compared with the more traditional applications of control theory, there are fundamentally new features introduced in this problem. The topology of the information network can have many effects. On one hand, it might introduce instability if the information being fed through the network adds on constructively to the disturbance at a node; on the other, intuitively, it should serve as a means for better noise rejection for the network as a whole. 
As a result of the above-mentioned properties, this problem has been garnering increasing attention. Fax and Murray [13], [14] obtained a Nyquist-like condition for stability of a formation using the individual plant transfer function and the Laplacian of the graph generated by the topology of the information flow network. Chaves et al. [6] considered the case of achieving a regular formation in vehicle networks for a milieu in which information is being lost stochastically. Jadbabaie et al. [20] considered the coordination of a group of autonomous agents when the graph topology changes over time and presented stability results for the case when the switching rule satisfi es certain properties. These results were expanded by Ren and Beard [27]. Gupta et al. [17] and Olfati-Saber and Murray [29] presented a general framework for decentralized analysis of stability of interconnected systems where the topology might potentially be time-varying.

However, most of the work so far has centered on stability analysis of the formation assuming certain control laws in place. A more general question is that of synthesis of the control law to be used by the agents in such a formation, such that some cost function is optimized. The defining feature of the problem is that while the cost function can involve all the individual agents in the formation; the pre-specified topology of the formation imposes constraints on the form of the control law by limiting the information available to various agents at any time. Thus, it is not realistic to assume that an agent would know the state of all the other agents in the formation at any given time and be able to use it to calculate the control input. These features make the problem a decentralized control problem with arbitrary information flow patterns, which is, in general, much harder to solve than the traditional optimal control problem.

Research in decentralized control has a long history. Witsenhausen [34], [35] showed that under the decentralized information constraints, a linear controller might not be optimal and also that the cost function need not be convex in the controller variables. A discrete equivalent of Witsenhausen's counter-example was given in [26] where it was also shown that the problem of fi nding a stabilizing controller under the information pattern constraints is NP-complete. For particular information structures, the problem has been solved, e.g., see [12]. Some researchers have also studied this problem under the assumption of spatial invariance by using a multidimensional approach (e.g., see [2], [8]). Rotkowitz et al. [28] gave certain invariance conditions under which the problem retains the convex character. A different approach for solving the problem was 
inspired by the design of reduced-order controllers (e.g. [25]) and yielded numerical algorithms for solving the optimal linear control with arbitrary number of free parameters for the infi nite horizon case as in [30], [33]. In [18], Gupta et al. explored this algorithm for the case of vehicle formations and in particular proved that in this case, it was always possible to choose a feasible initial point. A similar algorithm can be applied to the fi nite horizon problem, as described by Anderson and Moore [1], but the computational diffi culties were pointed out in [22]. The vehicle formation problem was also considered in [10] where the $H_{2}$ synthesis problem was posed as an optimization problem and a sub-optimal solution presented. Bemporad et al [3] considered the constrainted LQR problem and came up with a numerical algorithm for the optimal piecewise affi ne controller. The algorithm was extended to the case of infi nite-time horizon by Grieder et al [16]. A convex approach towards synthesizing control laws for solving distributed averaging problems was given in [36]. Receding horizon control for the problem was explored by Dunbar and Murray [11] and also by Franco et al. [15]. A good survey of the attempts to solve the related fi xed order and the static output feedback problems can be found in [31], [5], [9] and the references therein.

In this paper, we set up the LQR problem for the control of a network of autonomous agents with a given information flow topology. Even if the dynamics of the agents are not coupled and the only coupling present is due to the cost function, the optimal control law, in general, requires every agent to use knowledge about every other agent. We impose the constraint of a linear control law that satisfi es a pre-specified topology in that any agent uses only the information about a prespecifi ed set of agents with which it can communicate. We solve for the optimal control law for a fi nite time horizon under these constraints. We see that computation of the optimal control is computationally prohibitive and provide a sub-optimal solution instead which is computationally tractable. This algorithm is the chief contribution of this paper.

The outline of the paper is as follows. We address a few mathematical preliminaries in the next section. Then we set up and solve the constrained controller synthesis problem. We see that calculating the optimal solution is computationally intensive and hence propose a simpler sub-optimal solution. Then we present examples to illustrate the concepts and the algorithm. We see that the loss in performance by choosing the sub-optimal algorithm is not huge. We end with conclusions and present some avenues for further work. 


\section{Mathematical Preliminaries And Notations}

By a network of interconnected dynamic agents, we mean a system of agents whose dynamics are not coupled but in which every agent can use the information from a prescribed set of other agents (called its out-neighbors) for calculating its control input. The flow of information is thus described by identifying the set of out-neighbors for each agent and is referred to as the information flow topology. Consider a network of $n$ agents. Together with the information flow topology, the network can be represented by a graph in which the agents are vertices and allowed communication links are edges. We refer to the agents variously as vertices, nodes or vehicles and the network as a graph or a formation.

Consider a graph with $n$ nodes, the vertex set $V=\left\{v_{i}\right\}_{i=1}^{n}$ and edge set $E(G)$. The adjacency matrix (see, e.g., [4] for more details) denoted by $A$ is a square matrix of size $n \times n$, defi ned as follows

$$
A_{i j}= \begin{cases}1 & v_{i} v_{j} \in E(G) \\ 0 & \text { otherwise. }\end{cases}
$$

If we denote the out-degree of node $v_{i}$ by $O_{i}$, then the degree matrix denoted by $D$ is defined to be a square matrix of size $n \times n$, such that

$$
D_{i j}= \begin{cases}O_{i} & i=j \\ 0 & \text { otherwise. }\end{cases}
$$

We defi ne the Laplacian of a graph by the following equation

$$
L=D-A \text {. }
$$

We denote the expectation of a random variable $X$ by $E[X]$. The covariance matrix of a random variable $X$ with zero mean is defi ned by $E\left[X X^{\prime}\right]$. It is always a positive semi-defi nite matrix.

The trace of a square matrix $X$, denoted by $\operatorname{trace}(X)$, is defi ned as the sum of its diagonal elements. It is known that the trace is also the sum of the eigenvalues of $X$. The trace operator satisfi es the following properties (assume $X, Y$ and $Z$ to be compatible matrices; $v$ is a column vector).

1) $\operatorname{trace}(X+Y)=\operatorname{trace}(X)+\operatorname{trace}(Y)$.

2) $\operatorname{trace}(X Y Z)=\operatorname{trace}(Z X Y)$. 
3) $E\left[v^{\prime} W v\right]=E\left[\operatorname{trace}\left(W v v^{\prime}\right)\right]$.

In the last equation if $W$ is a constant matrix, the right hand side can be further rewritten as $\operatorname{trace}\left(W E\left[v v^{\prime}\right]\right)$.

We denote the transpose of a matrix $X$ by $X^{\prime}$ or $X^{T}$. For two matrices $A$ and $B$, we write $A>B$ if $A-B$ is a positive defi nite matrix. For a matrix $m \times n$ matrix $X=\left[x_{i j}\right]$, the operation $\operatorname{vec}(X)$ results in a $m n \times 1$ column vector with elements

$$
\operatorname{vec}(X)=\left[\begin{array}{c}
x_{11} \\
x_{21} \\
\vdots \\
x_{m 1} \\
x_{12} \\
\vdots \\
x_{m n}
\end{array}\right] .
$$

The operation $A \otimes B$ denotes the Kronecker product (also called the direct product) between two matrices $A$ and $B$ (see [24] for details). It can be shown that for suitably dimensioned matrices $A, X$ and $B$,

$$
\operatorname{vec}(A X B)=\left(B^{\prime} \otimes A\right) \operatorname{vec}(X)
$$

\section{PRoblem Formulation}

Consider a formation of $n$ agents, in which the $i$-th agent evolves according to the equation

$$
x_{k+1}^{i}=\Phi x_{k}^{i}+\Gamma u_{k}^{i}+w_{k}^{i},
$$

where the control law $u_{k}^{i}$ is given by

$$
u_{k}^{i}=F_{k}^{i, 1} x_{k}^{i}+\sum_{\text {all out-neighbors } j} F_{k}^{i j, 2}\left(x_{k}^{i}-x_{k}^{j}\right) .
$$

Assume that the noise $w_{k}^{i}$ is zero-mean, Gaussian and white. On stacking the state $x^{i}$ of all the agents, we can obtain the system state vector $x$, whose evolution is described by

$$
\begin{aligned}
x_{k+1} & =(I \otimes \Phi) x_{k}+(I \otimes \Gamma) u_{k}+w_{k} \\
u_{k} & =\left(\operatorname{diag}\left(F_{k}^{i, 1}\right)+L_{\text {gen }, k}\right) x_{k},
\end{aligned}
$$


where $I$ is identity matrix of suitable dimensions and $\operatorname{diag}\left(F_{k}^{i, 1}\right)$ is a block diagonal matrix with $F_{k}^{i, 1}$,s along the diagonal and zero matrices elsewhere. The vectors $u_{k}$ and $w_{k}$ are obtained by stacking the control laws and the noises for the individual agents, respectively. $L_{\text {gen }, k}$ is a generalization of the Laplacian matrix of the graph and is formed as follows. Create the adjacency matrix $A$ for the network. Then replace each unity element that is at the $(i, j)$-th place by $-F_{k}^{i j, 2}$. Replace the diagonal element in the $i$-th row by a matrix which is the sum of the matrices $F_{k}^{i 1,1}$, $F_{k}^{i 2,1}, \cdots, F_{k}^{i(i-1)}, F_{k}^{i(i+1)}, \cdots, F_{k}^{i n, 1}$. Rest of the zero elements are replaced by zero matrices of appropriate dimensions. Note that the topological constraints on the form of control law are inherent in the structure of $L_{\mathrm{gen}, k}$.

We make the following assumptions before proceeding further:

1) The topology of the network is fi xed and given.

2) Moreover the topology is known by all the agents. If that is not the case, we might need to run a broadcast algorithm to exchange the topology information among the nodes.

3) The communication links are ideal when they exist.

4) Finally, for simplicity, we assume that the full state of the agents is observed and transmitted. The generalization to output feedback is easily done.

Note that if all the vehicles are not identical, equations similar to (2) can easily be obtained. The matrices $I \otimes \Phi$ and $I \otimes \Gamma$ will be replaced by block diagonal matrices $\operatorname{diag}\left(\Phi^{i}\right)$ and $\operatorname{diag}\left(\Gamma^{i}\right)$, but other details remain similar. We begin by discussing the questions of stabilizability and controllability of the formation under a specifi ed topology constraint.

\section{A. Stabilizability}

Two questions arise immediately:

- Is it possible to stabilize a formation using information from other vehicles when the vehicles are individually not stable. In other words, if a vehicle is unstable, can the formation be stabilized by the exhange of information between different agents?

- Are some topologies inherently unstable in that even if the agents are stable, the information flow will always make it impossible to stabilize the formation?

We note the following result originally presented in [18].

Proposition 1. Consider a formation of interconnected dynamic agents as defined in section II. 
1) A formation is controllable if and only if each individual agent is controllable.

2) A formation is stabilizable if and only if each individual agent is stabilizable.

Proof: We use the notation introduced above. Let the matrix $\Phi$ be in $\mathbf{R}^{m}$ and there be $n$ agents in the formation. As can be seen from (2), for controllability of the formation, we want the following matrix to have rank $m n$ ([23])

$$
M_{1}=\left[\begin{array}{llll}
I \otimes \Gamma \quad(I \otimes \Phi)(I \otimes \Gamma) & (I \otimes \Phi)^{2}(I \otimes \Gamma) & \cdots & (I \otimes \Phi)^{m n-1}(I \otimes \Gamma)
\end{array}\right] .
$$

Using the standard property of Kronecker product

$$
(a \otimes b)(c \otimes d)=a c \otimes b d,
$$

we can rewrite $M_{1}$ as

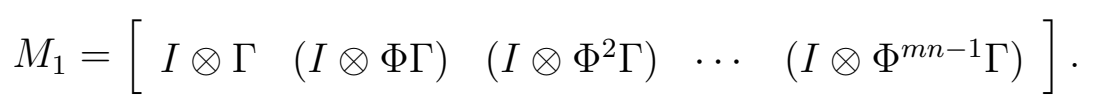

This matrix has rank $m n$ if and only if the following matrix has rank $m$

$$
M_{2}=\left[\begin{array}{lllll}
\Gamma & \Phi \Gamma & \Phi^{2} \Gamma & \cdots & \Phi^{m n-1} \Gamma
\end{array}\right] .
$$

Since $\Phi \in \mathbf{R}^{m}$, the equivalent condition is that the matrix

$$
M_{3}=\left[\begin{array}{lllll}
\Gamma & \Phi \Gamma & \Phi^{2} \Gamma & \cdots & \Phi^{m-1} \Gamma
\end{array}\right],
$$

be rank $m$. But $M_{3}$ being rank $m$ is simply the condition for the individual agent being controllable. Thus the formation is controllable if and only if each individual agent is controllable. This proves the first part. The second part also follows from the above proof. The subspace not spanned by the columns of $M_{1}$ is stable if and only if the subspace not spanned by the columns of $M_{3}$ is stable.

\section{B. Designing the Control Law}

From (2), it can be seen that the problem of designing a control law under the topological constraints is equivalent to solving the control design problem for the system

$$
\begin{aligned}
x_{k+1} & =(I \otimes \Phi) x_{k}+(I \otimes \Gamma) u_{k}+w_{k} \\
u_{k} & =F_{k} x_{k},
\end{aligned}
$$


with the additional constraint that $F_{k}$ should have those elements as 0 which correspond to zero entries in the $L_{\mathrm{gen}, k}$ of the interconnection topology formed as above. $F_{k}$ can then readily be cast in the form $\operatorname{diag}\left(F_{k}^{i, 1}\right)+L_{\mathrm{gen}, k}$ and the matrices $F_{k}^{i, 1}$ and $F_{k}^{i j, 2}$ obtained. It is fairly obvious that constraining the control $F_{k}$ to have some elements zero forces us to consider only those matrices $F_{k}$ that live in a particular sub-space of the vector space of all matrices with the same dimensions as $F_{k}$. It may be noted that since each node knows the topology, it knows the subspace within which the matrix $F_{k}$ should lie. Thus every node can carry the calculation of the control law by any of the methods discussed later in parallel to obtain the control law for the whole system. Then it can extract the control law that it needs to apply. We now defi ne the cost function we are aiming to minimize and solve the problem of fi nding the optimal control law.

\section{The Optimal Constrained Control LaW}

Denote $A=I \otimes \Phi$ and $B=I \otimes \Gamma$ and rewrite (3) as

$$
\begin{aligned}
x_{k+1} & =A x_{k}+B u_{k}+w_{k} \\
u_{k} & =F_{k} x_{k},
\end{aligned}
$$

where the initial condition $x_{0}$ is random and is Gaussian with zero-mean and covariance $R_{0}$. The noise $w_{k}$ is also random white zero-mean Gaussian with covariance $R_{w}$. In the above equations, $x_{k} \in \mathbf{R}^{n}$ is the state of the system and $u_{k} \in \mathbf{R}^{m}$ is the control input. We wish to minimize the cost function

$$
J_{T}=E\left[\sum_{k=0}^{T}\left\{x_{k}^{\prime} Q x_{k}+u_{k}^{\prime} R u_{k}\right\}\right]+E\left[x_{T+1}^{\prime} P_{T+1}^{c} x_{T+1}\right],
$$

where $Q$ and $R$ are positive defi nite. This is the classical LQR design problem. We can fi nd the optimal control law through solving the discrete-time Riccati recursion. Suppose we now wish to additionally constrain the control law to lie within a space spanned by the basis vectors $\left\{\Lambda_{j}\right.$, $j=1,2, \ldots, N\}$. Thus the problem is to find a control law of the form

$$
F_{k}=\sum_{j=1}^{N} \alpha_{k}^{j} \Lambda^{j}
$$

where $\alpha_{k}^{j}$ 's are scalars, that minimizes the cost function (5). 


\section{Remarks:}

1) It is fairly obvious that the optimal constrained control law would not, in general, be the projection of the optimal control law on to the sub-space we are interested in. This is reminiscent of the fact that the optimal causal estimate for a random variable is not the same as the projection of the general optimal estimate on to the causal sub-space [21].

2) Requiring apriori that the controller be linear might be a non-trivial assumption. But this allows us to derive algorithms for solving the problem and leads to sharper results.

\section{A. Preliminary Result}

In this subsection we prove an intermediate result that we will use later. First note the following lemma.

Lemma 2. Suppose $W$ is positive semi-definite and $P(K)$ denote any matrix-valued function of the matrix argument $K$. If $P(K)>P\left(K_{0}\right)$, then trace $(P(K) W) \geq \operatorname{trace}\left(P\left(K_{0}\right) W\right)$.

Proof: Since $P(K)>P\left(K_{0}\right)$, we have $P(K)-P\left(K_{0}\right)>0$. Also $W$ is positive semidefinite, thus $W^{\frac{1}{2}}$ is defined. Hence we note that $\operatorname{trace}\left(W^{\frac{T}{2}}\left(P(K)-P\left(K_{0}\right)\right) W^{\frac{1}{2}}\right)>0$ or that trace $\left(\left(P(K)-P\left(K_{0}\right)\right) W\right)>0$. But this means trace $(P(K) W)>\operatorname{trace}\left(P\left(K_{0}\right) W\right)$, which proves the assertion.

Using this lemma we can prove the following.

Proposition 3. Consider the cost function

$$
C=E\left[\left[\begin{array}{c}
K_{1} Y_{1}-X_{1} \\
K_{2} Y_{2}-X_{2} \\
\vdots \\
K_{n} Y_{n}-X_{n}
\end{array}\right]^{\prime} W\left[\begin{array}{c}
K_{1} Y_{1}-X_{1} \\
K_{2} Y_{2}-X_{2} \\
\vdots \\
K_{n} Y_{n}-X_{n}
\end{array}\right]\right]
$$

where $K_{i}$ 's are arbitrary matrices while $Y_{i}$ 's and $X_{i}$ 's are vectors of suitable dimensions such that the cost function $C$ is well-defined. Suppose that $W$ can be written in the form

$$
W=\left[\begin{array}{lrrr}
W_{1,1} & W_{1,2} & \ldots & W_{1, n} \\
W_{2,1} & & \ldots & W_{2, n} \\
\vdots & & \ddots & \vdots \\
W_{n, 1} & W_{n, 2} & \ldots & W_{n, n}
\end{array}\right]
$$


where the blocks $W_{i, j}$ are of appropriate sizes so that the product $X_{i}^{\prime} W_{i, j} X_{j}$ is well defined and that $W$ is symmetric and positive definite. Then the optimal $K_{i}$ 's minimizing the cost function are given by the solution to the coupled matrix equations

$$
K_{j}=W_{j, j}^{-1}\left[\sum_{i} W_{j, i} R_{X_{i} Y_{j}}-\sum_{i \neq j} W_{j, i} K_{i} R_{Y_{i} Y_{j}}\right] R_{Y_{j}}^{-1}, \quad \forall j=1,2, \cdots, n,
$$

where $R_{Y_{i} Y_{j}}=E\left[Y_{i} Y_{j}^{\prime}\right]$ and $R_{X_{i} Y_{j}}=E\left[X_{i} Y_{j}^{\prime}\right]$.

Proof: For each $j$, we can write the terms depending on $K_{j}$ as

$$
C_{j}=\operatorname{trace}\left(K_{j} R_{Y_{j}} K_{j}^{\prime} W_{j, j}-K_{j} \Psi-\Psi^{\prime} K_{j}^{\prime}\right),
$$

where

$$
\Psi=\left[\sum_{i} R_{Y_{j} X_{i}} W_{i, j}-\sum_{i \neq j} R_{Y_{j} Y_{i}} K_{i}^{\prime} W_{i, j}\right] .
$$

Thus $K_{j}$ needs to be chosen so as to minimize $C_{j}$. The minimization is of the form

$$
\min _{X} \operatorname{trace}\left(X A X^{\prime} B+X C+C^{\prime} X^{\prime}\right)
$$

where $B$ is invertible and positive defi nite. This can be rewritten as

$$
\begin{aligned}
\min _{X} \operatorname{trace}\left(X A X^{\prime} B+X C+C^{\prime} X^{\prime}\right) & =\min _{X} \operatorname{trace}\left(X A X^{\prime} B+X C B^{-1} B+C^{\prime} X^{\prime} B B^{-1}\right) \\
& =\min _{X} \operatorname{trace}\left(X A X^{\prime} B+X C B^{-1} B+B^{-1} C^{\prime} X^{\prime} B\right) \\
& =\min _{X} \operatorname{trace}\left(\left(X A X^{\prime}+X C B^{-1}+B^{-1} C^{\prime} X^{\prime}\right) B\right) .
\end{aligned}
$$

Now we use lemma 2. Thus our problem reduces to that of determining $X$ such that $X A X^{\prime}+$ $X C B^{-1}+B^{-1} C^{\prime} X^{\prime}$ is minimized. We complete the squares to write

$$
X A X^{\prime}+X C B^{-1}+B^{-1} C^{\prime} X^{\prime}=\left(X+B^{-1} C^{\prime} A^{-1}\right) A\left(X+B^{-1} C^{\prime} A^{-1}\right)^{\prime}-B^{-1} C^{\prime} A^{-1} C .
$$

Thus the minimizing $X=-B^{-1} C^{\prime} A^{-1}$. Applying this to our original problem of determining $K_{j}$, we see that

$$
K_{j}=W_{j, j}^{-1}\left[\sum_{i} W_{j, i} R_{X_{i} Y_{j}}-\sum_{i \neq j} W_{j, i} K_{i} R_{Y_{i} Y_{j}}\right] R_{Y_{j}}^{-1} .
$$

This completes the proof. 
Note that for calculation of the $K_{j}$ 's, we can use the identity (1). Thus we obtain for each $K_{j}$, the equation

$$
\operatorname{vec}\left(K_{j}\right)=\operatorname{vec}\left(W_{j, j}^{-1} \sum_{i} W_{j, i} R_{X_{i} Y_{j}}\right)-\sum_{i \neq j}\left[\left(R_{Y_{i} Y_{j}} R_{Y_{j}}^{-1}\right)^{\prime} \otimes\left(W_{j, j}^{-1} W_{j, i}\right) \operatorname{vec}\left(K_{i}\right)\right] .
$$

We have one such equation for each $K_{j}, j=1, \ldots, n$. These equations can readily be solved to obtain the values of $\operatorname{vec}\left(K_{j}\right)$ and from them the matrices $K_{j}$ can easily be determined.

\section{B. The Optimal Control Law}

From (5) we see that the cost function to be minimized is

$$
J_{T}=E\left[\sum_{k=0}^{T} u_{k}^{\prime} R u_{k}+\sum_{k=0}^{T} x_{k}^{\prime} Q x_{k}\right]+E\left[x_{T+1}^{\prime} P_{T+1}^{c} x_{T+1}\right] .
$$

Using the equation

$$
x_{k}=A^{k} x_{0}+\sum_{j=0}^{k-1} A^{j} B u_{k-1-j}+\sum_{j=0}^{k-1} A^{j} w_{k-1-j}
$$

and the fact that the noise $w_{k}$ is white and zero-mean allows us to rewrite the cost function in the form

$$
J_{T}=E\left[\Gamma^{\prime} \mathbf{F} \Gamma+\Gamma^{\prime} \mathbf{G} \Lambda+\Lambda^{\prime} \mathbf{G}^{\prime} \Gamma+\Lambda^{\prime} \mathbf{H} \Lambda\right] .
$$

In the above equation

$$
\Gamma=\left[\begin{array}{lllll}
x_{0}^{\prime} & w_{0}^{\prime} & w_{1}^{\prime} & \cdots & w_{T}^{\prime}
\end{array}\right]^{\prime}
$$

is the vector of all the random variables involved. Similarly,

$$
\Lambda=\left[\begin{array}{llll}
u_{0}^{\prime} & u_{1}^{\prime} & \cdots & u_{T}^{\prime}
\end{array}\right]^{\prime}
$$

is the control vector that is the optimization variable, and the matrices $\mathbf{F}, \mathbf{G}$ and $\mathbf{H}$ are functions of $A, B, R, Q$ and $P_{T+1}^{c}$. The additional constraint on $\Lambda$ is that it has to be of the form

$$
\Lambda=\left[\begin{array}{c}
u_{0} \\
u_{1} \\
\vdots \\
u_{T}
\end{array}\right]=\left[\begin{array}{c}
F_{0} x_{0} \\
F_{1} x_{1} \\
\vdots \\
F_{T} x_{T}
\end{array}\right]
$$


where the matrices $F_{i}$ have some pre-specifi ed elements zero. In particular, if we write

$$
F_{i} x_{i}=\left[\begin{array}{c}
F_{i}^{1} x_{i} \\
F_{i}^{2} x_{i} \\
\vdots \\
F_{i}^{n} x_{i}
\end{array}\right]
$$

where $F_{i}^{j}$ is the control law applied by the $j$-th agent at time step $i$, then those elements of $F_{i}^{j}$ are zero that correspond to the elements in the state vector $x_{i}$ that the $j$-th agent does not have access to. We can pull the constraints into the state vector and write

$$
F_{i}^{j} x_{i}=K_{i}^{j} y_{i}^{j}
$$

where $K_{i}^{j}$ is now a matrix free of any constraints on its elements while the vector $y_{i}^{j}$ is a stacked vector of the states of the agents that the $j$-th agent has access to. This allows us to write

$$
F_{i} x_{i}=\left[\begin{array}{c}
K_{i}^{1} y_{i}^{1} \\
K_{i}^{2} y_{i}^{2} \\
\vdots \\
K_{i}^{n} y_{i}^{n}
\end{array}\right] .
$$

Thus $\Lambda$ can be written as

$$
\Lambda=\left[\begin{array}{c}
F_{0} x_{0} \\
F_{1} x_{1} \\
\vdots \\
F_{T} x_{T}
\end{array}\right]=\left[\begin{array}{c}
K_{0}^{1} y_{0}^{1} \\
K_{0}^{2} y_{0}^{2} \\
\vdots \\
K_{0}^{n} y_{0}^{n} \\
K_{1}^{1} y_{1}^{1} \\
\vdots \\
K_{1}^{n} y_{1}^{n} \\
\vdots \\
K_{T}^{0} y_{T}^{0} \\
\vdots \\
K_{T}^{n} y_{T}^{n}
\end{array}\right] .
$$


The $n T$ matrices $K_{i}^{j}$ are arbitrary and are the optimization variables. Now from (7), we see that the cost function can be written as

$$
\begin{aligned}
J_{T} & =E\left[\Gamma^{\prime} \mathbf{F} \Gamma+\Gamma^{\prime} \mathbf{G} \Lambda+\Lambda^{\prime} \mathbf{G}^{\prime} \Gamma+\Lambda^{\prime} \mathbf{H} \Lambda\right] \\
& =E\left[\left(\Lambda+H^{-1} G^{\prime} \Gamma\right)^{\prime} H\left(\Lambda+H^{-1} G^{\prime} \Gamma\right)\right]+E\left[\Gamma^{\prime}\left(G H^{-1} G^{\prime}+F\right) \Gamma\right] .
\end{aligned}
$$

The choice of $\Lambda$ only affects the first term. Thus the optimization problem is

$$
\min _{\Lambda} E\left[\left(\Lambda+H^{-1} G^{\prime} \Gamma\right)^{\prime} H\left(\Lambda+H^{-1} G^{\prime} \Gamma\right)\right]
$$

where $\Lambda$ is of the form (8). But this optimization problem is exactly in the form of Proposition 3. Thus we can optimize the value of the cost function. This solves the optimal control law problem.

Remarks:

1) The solution involves the calculation of second order statistic terms which can be calculated off-line since the topology of the network is assumed to be known.

2) The procedure holds even for the case when the topology is time-varying, as long as all the agents know the topology.

3) However note that we need to solve a total of $n T$ coupled matrix equations. This is a formidable computational burden. In the next subsection, we present a method that is computationally more tractable at the expense of being sub-optimal.

\section{A Sub-optimal Control Law Algorithm}

Once again we note from (5) that the $T$-horizon cost function to be minimized is

$$
J_{T}=E\left[\sum_{k=0}^{T} u_{k}^{\prime} R u_{k}+\sum_{k=0}^{T} x_{k}^{\prime} Q x_{k}\right]+E\left[x_{T+1}^{\prime} P_{T+1}^{c} x_{T+1}\right] .
$$

We need to choose $u_{0}, u_{1}, \cdots, u_{T}$ that minimize $J_{T}$. Following [19], we gather terms that depend on the choice of $u_{K}$ and $x_{K}$ and write them as

$$
\begin{aligned}
\Upsilon_{T} & =E\left[u_{T}^{\prime} R u_{T}+x_{T}^{\prime} Q x_{T}\right]+E\left[x_{T+1}^{\prime} P_{T+1}^{c} x_{T+1}\right] \\
& =E\left[\left[\begin{array}{ll}
u_{T}^{\prime} & x_{T}^{\prime}
\end{array}\right] \Delta\left[\begin{array}{c}
u_{T} \\
x_{T}
\end{array}\right]\right]+E\left[w_{T}^{\prime} P_{T+1}^{c} w_{T}\right] \\
& =S_{T}+O_{T}
\end{aligned}
$$


where

$$
\begin{aligned}
\Delta & =\left[\begin{array}{cr}
R+B^{\prime} P_{T+1}^{c} B & B^{\prime} P_{T+1}^{c} A \\
A^{\prime} P_{T+1}^{c} B & Q+A^{\prime} P_{T+1}^{c} A
\end{array}\right] \\
S_{T} & =E\left[\left[\begin{array}{ll}
u_{T}^{\prime} & x_{T}^{\prime}
\end{array}\right] \Delta\left[\begin{array}{l}
u_{T} \\
x_{T}
\end{array}\right]\right] \\
O_{T} & =E\left[w_{T}^{\prime} P_{T+1}^{c} w_{T}\right] .
\end{aligned}
$$

In the above equation, we have used the system dynamics given in (4) and the fact that the plant noise is zero mean. Thus we can write

$$
J_{T}=E\left[\sum_{k=0}^{T-1} u_{k}^{\prime} R u_{k}+\sum_{k=0}^{T-1} x_{k}^{\prime} Q x_{k}\right]+S_{T}+O_{T} .
$$

We aim to choose $u_{T}$ to minimize $J_{T}$. From (9), it is clear that the only term where the choice of $u_{T}$ can make a difference is $S_{T}$. On completing squares, $S_{T}$ can be written as

$$
S_{T}=E\left[\left(u_{T}-\bar{u}_{T}\right)^{\prime} R_{e, T}^{c}\left(u_{T}-\bar{u}_{T}\right)\right]+E\left[x_{T}^{\prime} P_{T}^{c} x_{T}\right]
$$

where

$$
\begin{aligned}
R_{e, T}^{c} & =R+B^{\prime} P_{T+1}^{c} B \\
P_{T}^{c} & =Q+A^{\prime} P_{T+1}^{c} A-A^{\prime} P_{T+1}^{c} B\left(R+B^{\prime} P_{T+1}^{c} B\right)^{-1} B^{\prime} P_{T+1}^{c} A
\end{aligned}
$$

and $\bar{u}_{T}$ is the standard optimal LQ control given by

$$
\bar{u}_{T}=-\left(R_{e, T}^{c}\right)^{-1} B^{\prime} P_{T+1}^{c} A x_{T} .
$$

If the controller had access to the entire state, it could simply use the standard optimal control $\bar{u}_{T}$. However, that is not possible now. Instead, the controller needs to calculate $u_{T}$ using the information flow that satisfi es the topological constarints and choose it to minimize $S_{T}$. In other words, we need to find $u_{T}=F_{T} x_{T}$ that minimizes $\Upsilon_{T}$ where $F_{T}$ has certain elements zero. The control problem thus reduces to an optimal estimation problem. Once again, we note that

$$
u_{T}=\left[\begin{array}{c}
u_{T}^{1} \\
u_{T}^{2} \\
\vdots \\
u_{T}^{n}
\end{array}\right],
$$


where each $u_{T}^{i}$ is the control law the $i$-th agent applies and it is a linear function of the measurements the $i$-th agent has access to. Thus we can write

$$
u_{T}^{i}=F_{T}^{i} x_{T}
$$

where $F_{T}^{i}$ has those elements 0 that correspond to the elements in the state vector $x_{T}$ that the $i$-th agent does not have access to. Pulling the constraints into the state vector, we can write

$$
u_{T}^{i}=K_{T}^{i} y_{T}^{i}
$$

where $K_{T}^{i}$ does not have any constraint while the vector $y_{T}^{i}$ is a stacked vector of the states of the agents that the $i$-th agent has access to. Thus the problem of choosing the control law $u_{T}$ reduces to the problem of choosing $K_{T}^{i}$ 's so as to minimize the criterion

$$
E\left[\left[\begin{array}{c}
K_{T}^{1} y_{T}^{1}-\bar{u}_{T}^{1} \\
K_{T}^{2} y_{T}^{2}-\bar{u}_{T}^{2} \\
\vdots \\
K_{T}^{n} y_{T}^{n}-\bar{u}_{T}^{n}
\end{array}\right]^{\prime} R_{e, T}^{c}\left[\begin{array}{c}
K_{T}^{1} y_{T}^{1}-\bar{u}_{T}^{1} \\
K_{T}^{2} y_{T}^{2}-\bar{u}_{T}^{2} \\
\vdots \\
K_{T}^{n} y_{T}^{n}-\bar{u}_{T}^{n}
\end{array}\right]\right] .
$$

This is exactly the optimization problem discussed in Proposition 3. Thus the matrices $K_{T}^{i}$ can be easily obtained. Note that this involves solving only $n$ coupled matrix equations and is hence much less computationally expensive than the optimal control law calculation discussed in section IV-B.

Denote the estimation error incurred due to the minimizing choice of $u_{T}$ by $\Lambda_{T}$. We have

$$
S_{T}=\Lambda_{T}+E\left[x_{T}^{\prime} P_{T}^{c} x_{T}\right]
$$

We can thus write the cost function as

$$
\begin{aligned}
J_{T} & =E\left[\sum_{k=0}^{T-1} u_{k}^{\prime} R u_{k}+\sum_{k=0}^{T-1} x_{k}^{\prime} Q x_{k}\right]+S_{T}+O_{T} \\
& =E\left[\sum_{k=0}^{T-1} u_{k}^{\prime} R u_{k}+\sum_{k=0}^{T-1} x_{k}^{\prime} Q x_{k}\right]+\Lambda_{T}+E\left[x_{T}^{\prime} P_{T}^{c} x_{T}\right]+O_{T} \\
& =J_{T-1}+\Lambda_{T}+O_{T}
\end{aligned}
$$

Thus we now need to choose control inputs for time steps 0 to $T-1$ to minimize $J_{T}$. By scanning the terms on the right hand side of the equation, we see that $O_{T}$ is independent of the choice of control laws from time 0 to $T-1$. However, unlike the standard case of control with imperfect 
observations [19], we note that apart from $J_{T-1}$, the estimation error $\Lambda_{T}$ is also a function of the state $x_{T}$ and hence of the (unknown) control law $u_{T-1}$. Moreover it is a non-linear function of $u_{T-1}$. Thus the control $u_{T-1}$ should be chosen to minimize the cost $J_{T-1}+\Lambda_{T}$. Thus, the separation principle does not hold in general. This is related to the fact that the information pattern is not classical (see, e.g., [35]) because the previous control law is not known fully to all the agents. We get across this problem by neglecting the estimation cost $\Lambda_{T}$ and optimizing only $J_{T-1}$. For this purpose, we note that our argument so far was independent of time index $T$. Thus we can recursively apply the argument for time steps $T-1, T-2$ and so on.

\section{Remarks:}

1) We have enforced a separation principle artifi cially that says that the controller synthesis problem can be separated into an estimation problem and the usual LQR control problem. At every time step, every controller tries to estimate the optimal control law from the information it has access to (in the sense of Proposition 3) and uses this estimate in the optimal LQR control law.

2) This method is in general sub-optimal since the separation principle does not hold in reality. However since this method replaces solution of $n T$ coupled matrix equations by solving $n$ coupled matrix equations $T$ times, this method saves a lot on computational cost.

3) If needed, better performance can be achieved by including the estimation cost $\Lambda_{T}$ in calculation of $u_{T-1}$. It can be proved that this inclusion results in a convex problem that can be solved effi ciently. However this method would still not be optimal since for calculation of $u_{T-2}$, we need to consider $J_{T-2}, \Lambda_{T-1}$ and the cost incurred in imperfectly minimizing $\Lambda_{T}$. Thus the problem starts involving more and more terms to optimize over. The extent of sub-optimality can be reduced by including more terms in the optimization.

4) Intuitively, the approximation can be thought of as follows. At any time, the optimal control input of an agent will depend on the control inputs of other agents at the previous time step. However the agent is not allowed to observe these. We get around this problem by ignoring the direct dependence of the optimal control input on these terms. Instead, we use the fact that these terms will soon show up in the values of the states of the neighbors of the agent, which are being observed. Thus these terms will eventually be used in the 
calculation of control inputs.

\section{EXAMPLES}

We now consider two examples to illustrate the issues involved.

Example 1: Consider a network of four agents, each with single integrator dynamics. This case is of interest since single integrator dynamics can be used to solve consensus problems. Let the agents be designated as $v_{i}, i=1,2,3,4$. The agent $v_{i}$ has dynamics

$$
\begin{aligned}
x_{k+1}^{i} & =x_{k}^{i}-0.2 u_{k}^{i}+w_{k}^{i} \\
u_{k}^{i} & =F^{i, 1} x_{k}^{i}+\sum_{\text {all out-neighbors } j} F^{i j, 2}\left(x_{k}^{j}-x_{k}^{i}\right) .
\end{aligned}
$$

We denote $x_{k}$ to be the state of the whole system, where

$$
x_{k}=\left[x_{k}^{1}, x_{k}^{2}, x_{k}^{3}, x_{k}^{4}\right]^{\prime} .
$$

Similarly denote $u_{k}$ to be the control vector obtained by stacking all the $u_{k}^{i}$,s. Then the evolution of the system is described as

$$
\begin{aligned}
x_{k+1} & =x_{k}-0.2 u_{k}+w_{k} \\
u_{k} & =F^{1} x_{k}+F^{2} x_{k},
\end{aligned}
$$

where $F^{1}$ is a diagonal matrix with $F^{1,1}, F^{2,1}, F^{3,1}, F^{4,1}$ as the diagonal elements; and the $(i, j)$-th element of the matrix $F^{2}$ is given by

$$
\left[F^{2}\right]_{i, j}= \begin{cases}F^{i j, 2} & i \neq j \text { and } j \text { is an out-neighbor of } i \\ 0, & i \neq j \text { and } j \text { is not an out-neighbor of } i \\ -\sum_{j} F^{i j, 2}, & i=j .\end{cases}
$$

The initial condition is random with zero mean and covariance as identity matrix. Similarly the noise is white Gaussian with zero mean and covariance as identity matrix. The cost function specifi ed is

$$
J=\sum_{k=0}^{T} E\left[x_{k}^{\prime} Q x_{k}+u_{k}^{\prime} R u_{k}\right] .
$$


We present results for $T=30$. We take the weighting matrices to be as follows:

$$
\begin{aligned}
Q & =\left[\begin{array}{cccc}
1.6158 & 1.6884 & 1.2138 & 0.563 \\
1.6884 & 2.798 & 1.2843 & 1.2528 \\
1.2138 & 1.2843 & 0.9645 & 0.5147 \\
0.563 & 1.2528 & 0.5147 & 0.7501
\end{array}\right] \\
R & =\left[\begin{array}{llll}
1 & 0 & 0 & 0 \\
0 & 1 & 0 & 0 \\
0 & 0 & 1 & 0 \\
0 & 0 & 0 & 1
\end{array}\right]
\end{aligned}
$$

First we note that if all the agents are communicating with one another, the sub-optimal and optimal algorithm give the same cost and the control law matrix. We also consider a constrained topology where we allow limited communication to happen. The topology is as follows. The vehicle $v_{1}$ can talk to $v_{2}$, the vehicle $v_{2}$ to $v_{1}$ and $v_{3}$, the vehicle $v_{3}$ to $v_{2}$ and $v_{4}$ and $v_{4}$ can talk to $v_{3}$. In this case, the evolution of the cost is shown in figure 1 . We can see that the loss in performance from the sub-optimal algorithm is not huge. The savings in computational time are considerable, however. Note that at the intermediate time values, the sub-optimal algorithm is performing better than the optimal algorithm. However, this can be easily explained by noting that the optimal algorithm is optimal for a time horizon of 30 steps and there is no guarantee that it is the optimal algorithm for a smaller time window as well.

In fi gure 2 we show the steady state cost for the ring topology for a time horizon of 100 time steps for the ring topology as we introduce delay into the system. The ring topology involves all communication links being present, except the $v_{2} v_{4}$ and $v_{1} v_{3}$ links. We assume that the state information is passed with some delay as a multiple of sampling time of the system but the agents calculate the control law assuming there is no delay. It can be seen that the cost slowly increases and the system is reasonably robust to delay uncertainity. It becomes unstable only for a delay equal to or greater than 5 time steps.

Example 2: In this example we use the dynamics of each agent as the dynamics of the Caltech Multi Vehicle Wireless Testbed vehicles, as described in [7], [32]. The non-linear dynamics are 


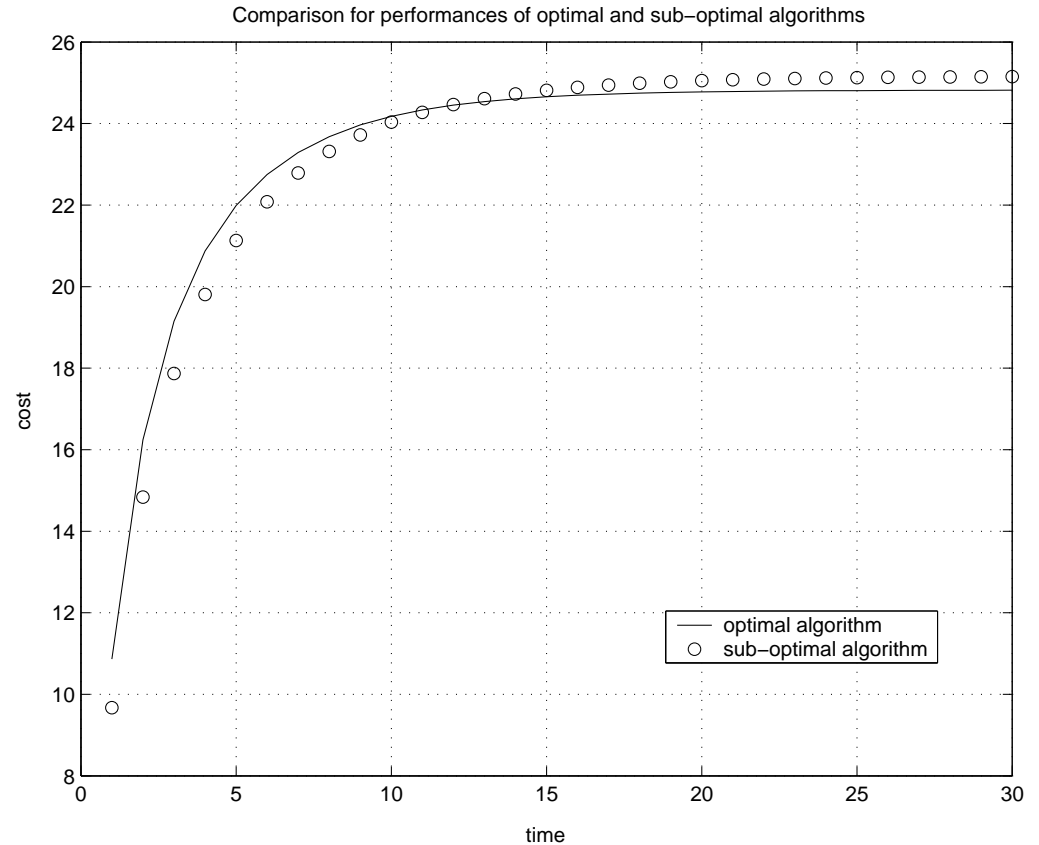

Fig. 1. The loss in performance due to the sub-optimal algorithm is not huge. Cost considered is $E\left[x_{k}^{\prime} Q x_{k}+u_{k}^{\prime} R u_{k}\right]$.

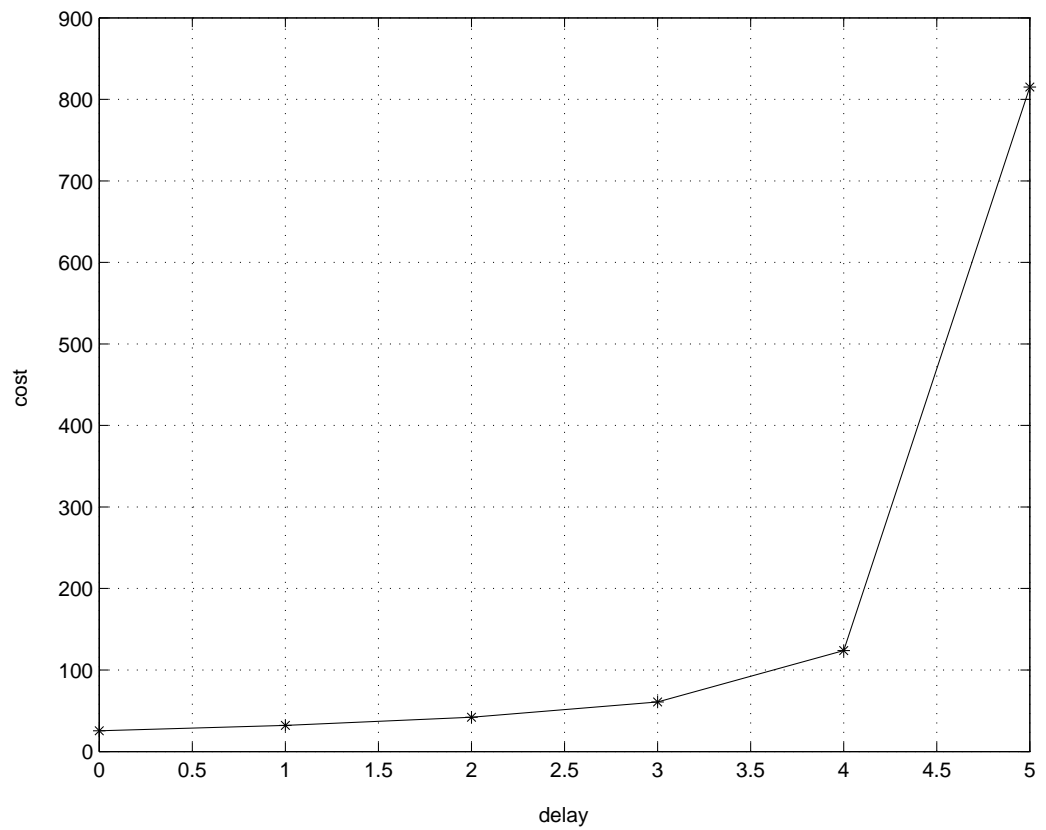

Fig. 2. The sub-optimal algorithm is robust to delays. Cost considered is $E\left[x_{k}^{\prime} Q x_{k}+u_{k}^{\prime} R u_{k}\right]$. 
given by

$$
\begin{aligned}
m \ddot{x} & =-\mu \dot{x}+\left(F_{L}+F_{R}\right) \cos (\theta) \\
m \ddot{y} & =-\mu \dot{y}+\left(F_{L}+F_{R}\right) \sin (\theta) \\
J \ddot{\theta} & =-\psi \dot{\theta}+\left(F_{R}-F_{L}\right) r_{f} .
\end{aligned}
$$

$F_{L}$ and $F_{R}$ are the inputs, $m=0.749 \mathrm{~kg}$ is the mass of vehicle, $J=0.0031 \mathrm{~kg} \mathrm{~m}^{2}$ is the moment of inertia, $\mu=0.15 \mathrm{~kg}$-s is the linear frictional coeffi cient, $\psi=0.005 \mathrm{kgm}^{2} / \mathrm{s}$ is the rotational friction coeffi cient and $r_{f}=0.089 \mathrm{~m}$ is the distance from the center of mass of the vehicle to the axis of the fan. On linearizing the dynamics about the straight line $y=x$ at a velocity of $1 \mathrm{~ms}^{-1}$ along the $\mathrm{x}$ and $\mathrm{y}$ axes, we obtain the equations

$$
\begin{aligned}
\dot{X} & =A X+B U \\
U & =F X
\end{aligned}
$$

where

$$
\begin{aligned}
& X=\left[\begin{array}{llllll}
x & y & \theta & \dot{x} & \dot{y} & \dot{\theta}
\end{array}\right]^{\prime} \\
& A=\left[\begin{array}{cccccr}
0 & 0 & 0 & 1 & 0 & 0 \\
0 & 0 & 0 & 0 & 1 & 0 \\
0 & 0 & 0 & 0 & 0 & 1 \\
0 & 0 & \frac{-\left(F_{L}^{n o m}+F_{R}^{n o m}\right) \sin \left(\theta^{n o m}\right)}{m} & \frac{-\mu}{m} & 0 & 0 \\
0 & 0 & \frac{\left(F_{L}^{n o m}+F_{R}^{n o m}\right) \cos \left(\theta^{n o m}\right)}{m} & 0 & \frac{\mu}{m} & 0 \\
0 & 0 & 0 & 0 & 0 & \frac{-\psi}{J}
\end{array}\right] \\
& B=\left[\begin{array}{lr}
0 & 0 \\
0 & 0 \\
0 & 0 \\
\frac{\cos \left(\theta^{n o m}\right)}{m} & \frac{\cos \left(\theta^{n o m}\right)}{m} \\
\frac{\sin \left(\theta^{n o m}\right)}{m} & \frac{\sin \left(\theta^{\text {nom }}\right)}{m} \\
\frac{-r_{f}}{J} & \frac{-r_{f}}{J}
\end{array}\right] \\
& \theta^{\text {nom }}=\frac{\pi}{4} \quad F_{L}^{\text {nom }}=F_{R}^{\text {nom }}=\frac{\mu}{\sqrt{2}} .
\end{aligned}
$$


We discretize the above equations with a step size $h=0.2$. We consider 8 vehicles starting from an octagonal formation and consider the topologies possible as the communication radius of each vehicle is increased. It is apparent that by symmetry there are 5 distinct topologies possible, with each vehicle talking to $0,2,4,6$ and 7 other vehicles respectively. The initial covariance matrix $R_{0}$ is the identity matrix. The cost function matrix $R$ is also identity while the matrix $Q$ is randomly generated. The cost function horizon is $T=100$ time steps. A typical curve for the varying of the costs provided by the sub-optimal algorithm as the communication radius is increased is given in fi gure 3. Following general conclusions can be drawn for the

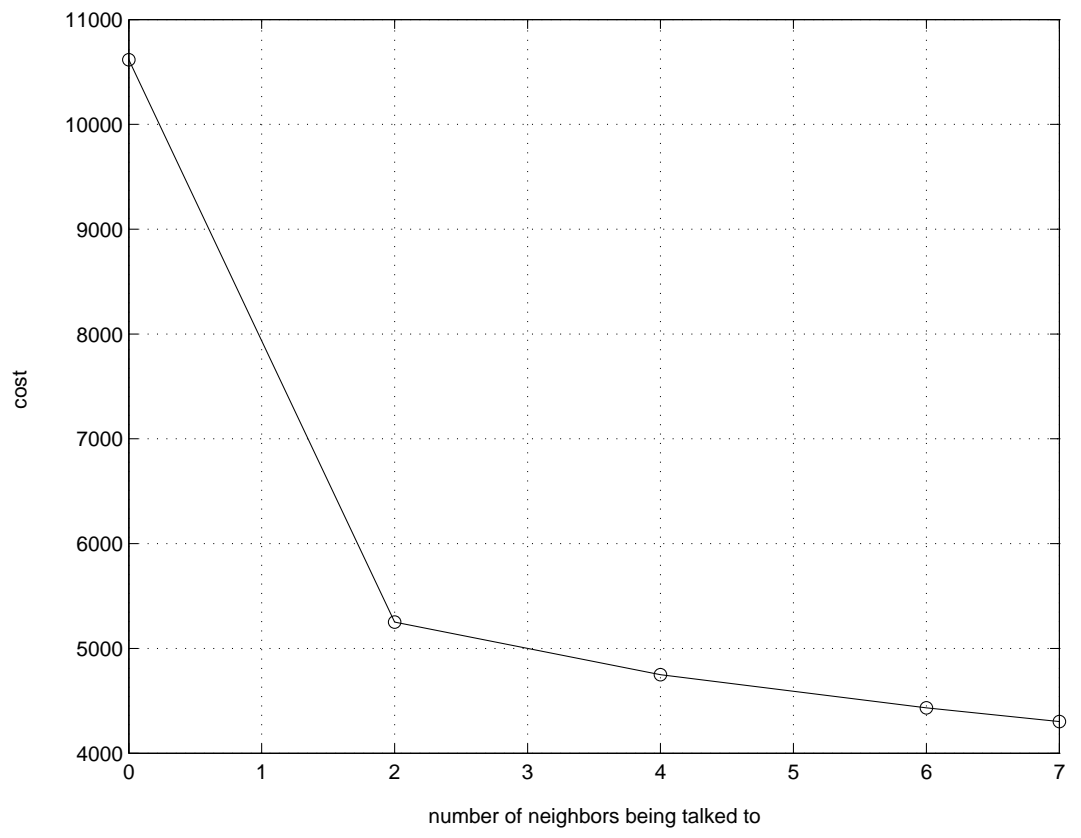

Fig. 3. As the communication radius is increased, the cost goes down. Cost considered is $E\left[x_{k}^{\prime} Q x_{k}+u_{k}^{\prime} R u_{k}\right]$.

example from the plot.

1) As more and more communication is allowed, the cost goes down.

2) The marginal utility of each communication link decreases as more and more links are added. However this might simply be due to the fact that the edges added later bring the data of far-away vehicles which is not so important for stabilization.

The difference in the performance between the sub-optimal and the optimal algorithms increased as the communication topology became more and more sparse. Figure 4 shows another plot 
comparing the comparison of optimal and sub-optimal algorithms for a different value of the $Q$ matrix. It can be seen that even for the decentralized case, the error is of the order of only $30 \%$.

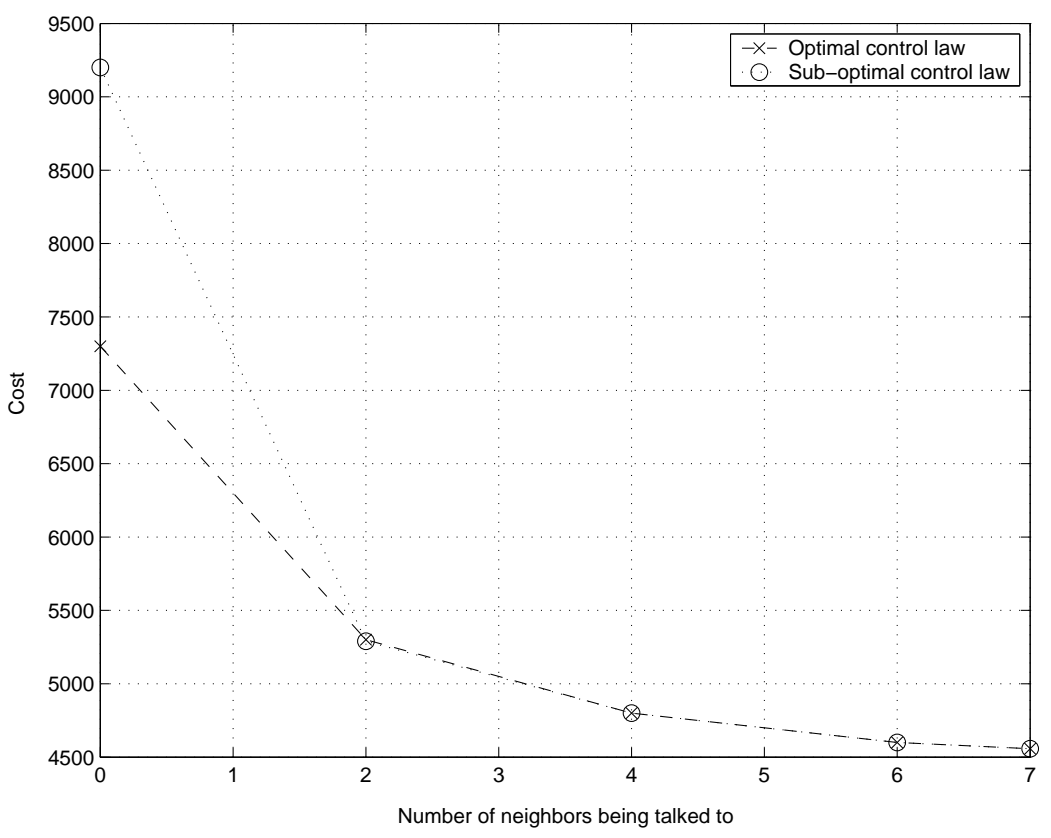

Fig. 4. As the communication radius is increased, loss in performance due to the sub-optimal algorithm decreases. Cost considered is $E\left[x_{k}^{\prime} Q x_{k}+u_{k}^{\prime} R u_{k}\right]$.

\section{CONCLUSIONS AND Future WORK}

In this paper, motivated by synthesis of optimal control laws for interconnected network of agents, we considered the problem of synthesis of a LQR optimal control law which is constrained to lie in a particular vector space. We constrained the controller to be linear. We presented a computationally expensive method for the optimal fi nite time horizon control and a computationally easier method to generate a sub-optimal control law. We presented examples which illustrated that the loss in performance due to the sub-optimal algorithm is not huge and that communication in general helps to bring down the cost.

The work can potentially be extended in many ways. The most obvious direction is to consider the case when the nodes do not have an accurate or complete knowledge of the entire network. It would be interesting if we could evaluate the cost if the nodes utilize the information 
corresponding only to the neighboring nodes. This would be especially useful in the case when topology is time-varying. The issue of optimal topology is also an interesting one to explore.

\section{Acknowledgments \\ The research was supported in part by AFOSR grant F49620-01-1-0460 and in part by NSF grant CCR-0326554.}

\section{REFERENCES}

[1] B. D. O. Anderson and J. B. Moore. Linear Optimal Control. Prentice-Hall, 1971.

[2] B. Bamieh, F. Paganini, and M. A. Dahleh. Distributed control of spatially invariant systems. IEEE Transactions on Automatic Control, 47(7):1091-1107, July 2002.

[3] A. Bemporad, M. Morari, V. Dua, and E. N. Pistikipoulos. The explicit linear quadratic regulator for constrained systems. Automatica, 38(1):3-20, January 2002.

[4] N. Biggs. Algebraic Graph Theory. Cambridge Tracks in Mathematics. Cambridge University Press, 1974.

[5] V. Blondel, M. Gevers, and A. Lindquist. Survey on the state of systems and control. European Journal of Control, 1:5-23, 1995.

[6] M. Chaves, R. Day, L. Gomez-Ramos, P. Nag, A. Williams, W. Zhang, and S. Glavaski. Vehicle networks: Achieving regular formation. Technical report, Report, 2002 IMA Summer Program, 2002.

[7] L. Cremean, B. W. Dunbar, D. van Gogh, J. Hickey, E. Klavins, J. Meltzer, and R. M. Murray. The Caltech multi-vehicle wireless testbed. In Proc of the 2002 Conference on Decision and Control, 2002.

[8] R. D’Andrea and G. E. Dullerud. Distributed control design for spatially interconnected systems. IEEE Transactions on Automatic Control, 48(9):1478-1495, 2003.

[9] S. Darbha, S. Pargaonkar, and S. P. Bhattacharya. A linear programming approach to the synthesis of fi xed structure controllers. In Proceedings of the 2004 American Control Conference, 2004.

[10] G. A. de Castro. Convex Methods for the design of Structured Controllers. PhD thesis, University of California, Los Angeles, 2003.

[11] W. B. Dunbar and R. M. Murray. Distributed receding horizon control with applications to multi-vehicle formation stabilization. Automatica. Submitted.

[12] C. Fan, J. L. Speyer, and C. R. Jaensch. Centralized and decentralized solutions of the linear-exponential-gaussian problem. IEEE Transactions on Automatic Control, 39(10):1986-2003, 1994.

[13] J. A. Fax. Optimal and Cooperative Control of Vehicle Formations. PhD thesis, California Institute of Technology, 2001.

[14] J. A. Fax and R. M. Murray. Information fbw and cooperative control of vehicle formations. IEEE Transactions on Automatic Control. submitted.

[15] E. Franco, T. Parisini, and M. Polycarpou. Cooperative control of discrete-time agents with delayed information exchange: a receding horizon approach. In Proceedings of the 2004 IEEE Control and Decision Conference, December 2004. To appear.

[16] P. Grieder, F. Borrelli, F. Torrisi, and M. Morari. Computation of the constrained infi nite time linear quadratic regulator. In Proc. of the American Control Conference, June 2003. 
[17] V. Gupta, B. Hassibi, and R. M. Murray. Stability analysis of stochastically varying formations of dynamic agents. In Proc. of IEEE Conf. on Decision and Control, 2003.

[18] V. Gupta, B. Hassibi, and R. M. Murray. On the synthesis of control laws for a network of autonomous agents. In Proc. of the American Control Conference, June 2004.

[19] B. Hassibi, A. H. Sayed, and T. Kailath. Indefi nite-Quadratic Estimation and Control. Studies in Applied and Numerical Mathematics, 1999.

[20] A. Jadbabaie, J. Lin, and A. S. Morse. Coordination of groups of mobile autonomous agents using nearest neighbor rules. IEEE Transactions on Automatic Control, 48(6):988-1001, 2003.

[21] T. Kailath, A. H. Sayed, and B. Hassibi. Linear Estimation. Prentice Hall, 2000.

[22] D. L. Kleinman, T. Fortmann, and M. Athans. On the design of linear systems with piecewise-constant feedback gains. IEEE Transactions on Automatic Control, AC-13(4):354-361, August 1968.

[23] H. Kwakernaak and R. Sivan. Linear Optimal Control Systems. John Wiley and Sons, 1972.

[24] P. Lancaster. Theory of Matrices. Academic Press, 1969.

[25] W. S. Levine, T. L. Johnson, and M. Athans. Optimal limited state variable feedback controllers for linear systems. IEEE Transactions on Automatic Control, AC-16:785-793, 1971.

[26] C. H. Papadimitriou and J. N. Tsitsiklis. Intractable problems in control theory. SIAM Journal on Control and Optimization, 24:639-654, 1986.

[27] W. Ren and R. W. Beard. Consensus of information under dynamically changing interaction topologies. In Proc. of the American Control Conference, pages 4939-4944, June 2004.

[28] M. Rotkowitz and S. Lall. Decentralized control information structures preserved under feedback. In Proc. of IEEE Conf. on Decision and Control, 2002.

[29] R. O. Saber and R. M. Murray. Consensus problems in networks of agents with switching topology and time-delays. IEEE Transactions on Automatic Control. Submitted.

[30] T. Soderstrom. On some algorithms for design of optimal constrained regulators. IEEE Transactions on Automatic Control, AC-23:1100-1101, 1978.

[31] V. L. Syrmos, C. T. Abdullah, P. Dorato, and K. Grigoriadis. Static output feedback - a survey. Automatica, 33(2):125-137, 1997.

[32] S. Waydo, Z. Jin, E. Wildanger, M. Lammers, H. Scholze, P. Foley, R. M. Murray, and D. Held. MVWT-II: The second generation caltech multi-vehicle wireless testbed. In American Control Conference, 2004.

[33] C. J. Wenk and C. H. Knapp. Parameter optimization in linear systema with arbitrarily constrained controller structure. IEEE Transactions on Automatic Control, AC-25(3):496-500, 1980.

[34] H. S. Witsenhausen. A counterexample in stochastic optimum control. SIAM Journal of Control, 6(1):131-147, 1968.

[35] H. S. Witsenhausen. Separation of estimation and control for discrete time systems. Proceedings of the IEEE, 59(11):1557$1566,1971$.

[36] L. Xiao and S. Boyd. Fast linear iterations for distributed averaging. System and Control Letters, 53:65-78, 2004. 